\title{
KNOWLEDGE AND SKiLl of PATIENTS WITH REGARD TO AMPUTATION STUMP BANDAGING, PRIOR TO A PROSTHESIS
}

\begin{abstract}
A swollen or poorly coned stump makes it impossible for the prosthetist to provide a patient who had an amputation with a prosthesis.

VISSER C

It is both costly, and time consuming for the patient and the amputation team if the patient needs to make repeated visits to the clinic. The purpose of this study is to determine the knowledge and skill of patients with regard to amputation stump bandaging. Thirty-three lower limb amputees, who visited the amputation clinic, were randomly chosen to participate in this survey. The investigation included a questionnaire and a physical evaluation by a physiotherapist on the effectiveness of the bandaging.

Results: Only 16 (49\%) of subjects received information, education and demonstration on bandaging for their stump. Three of the 16 subjects were judged to have had effective stump bandaging. From this study it is clear that there is a lack of knowledge and skills relating to bandaging amongst amputation patients and that this urgently needs to be rectified to ensure maximal functional outcomes.
\end{abstract}

\section{INTRODUCTION}

The reason why a primary amputee may be referred to the local amputation team is for cast, measurement, fitting and subsequent delivery of a prosthesis. Not every patient referred to the prosthetic rehabilitation team will be fitted with a prosthesis. The success of the rehabilitation program is determined to some extent by the individual's physical characteristics of the residual limb. A wellhealed, cylindrical limb with a nonadherent scar is easier to fit than one that is conical or has redundant tissue distally or laterally (O'Sullivan and Schmitz 1988). Prosthetists at the Pretoria Academic Hospital (PAH) are concerned about the quality of amputation stump shape and size of patients presenting at the amputation clinic for a prosthesis.

At the PAH, patients attend the amputation clinic six weeks after the amputation for the first cast model in preparation for a prosthesis to be made. If oedema is still present at this stage, prosthetic fitting will be delayed (Brady 1982). Control and reduction of oedema are prerequisites for wound healing, coning and eventual prosthetic fitting (MacLean and Fick 1994). Soft dressings remain a popular form of post-operative dressing technique for the amputee. Sustainable pressures greater than $15 \mathrm{~mm} \mathrm{Hg}$ have been found to decrease blood flow and sustained pressures greater than $25 \mathrm{~mm} \mathrm{Hg}$ have been found to be potentially harmful, due to tissue ischemia as a result of

CORRESPONDENCE:

Mrs C Visser

(Lecturer University of Pretoria)

B Phys T (UP)

PO Box 710 Faerie Glen 0043 Tel (h) (012) 9911953 (w) (012) 3542023 direct mechanical pressure (Spiro et al 1980). Intercapillary pressure varies with dependency of the limb, therefore the ideal bandage should provide graded pressure that is maximal at the most distal point and decreases proximally (Isherwood et al 1975). Isherwood et al (1975) compared pressures under amputee dressings that had been applied by skilled staff to those applied by unskilled staff. The conclusion was made that elastic bandages can be potentially dangerous when applied by unskilled individuals. Soft dressings remain popular, as they are inexpensive, readily available, quick to apply and frequent observations to the wound are possible (Browse 1974).
Bandaging is an acquired skill. If applied correctly, it is an effective and economical stump shrinkage tool (O'Sullivan and Schmitz 1988). The bandaging must be done accurately, supervised continuously, and taught to the amputee (Holliday 1981). Early prothetic limb fitting not only hastens recovery of an amputation but can be cost effective and time saving (Pinzur et al 1988).

Since bandaging is so vital, this study was initiated to determine the knowledge and skill of patients with regard to amputation stump bandaging, prior to fitting of a prosthesis. This study will also attempt to provide solutions for the possible lack of knowledge and skills by means of the

\section{FIGURE 1}

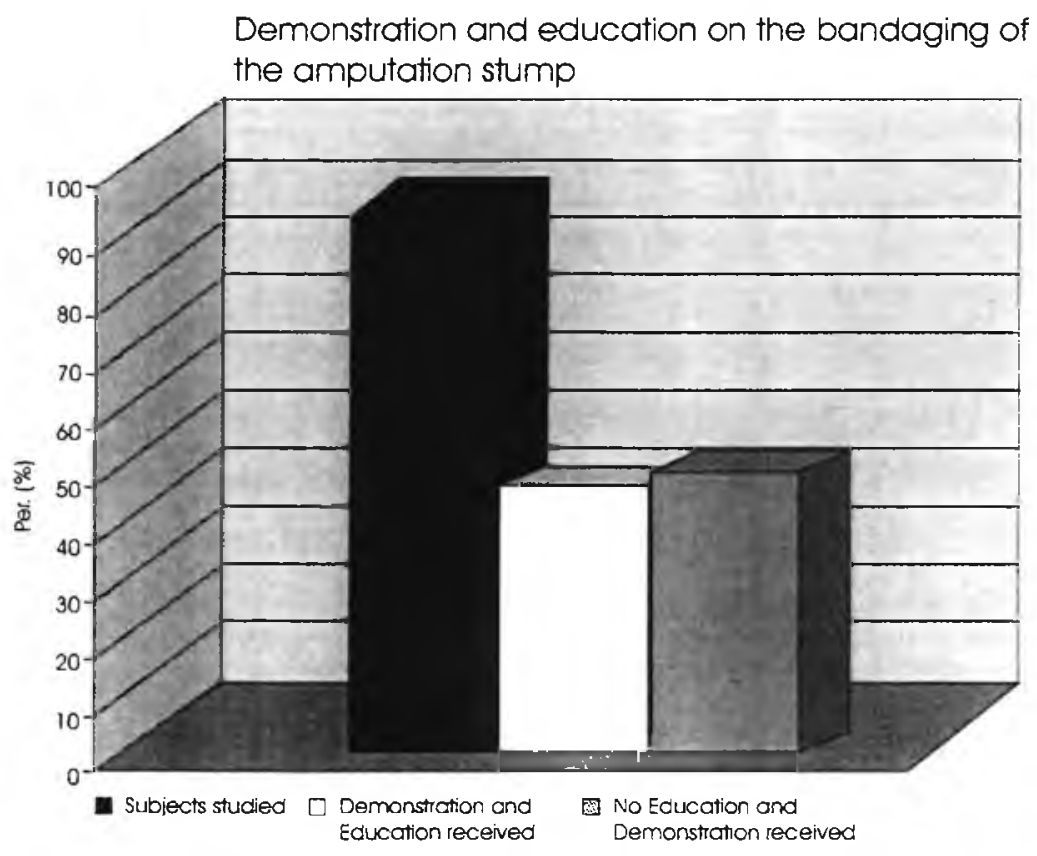

Fig 1: Demonstration and education on the bandaging of the amputation stump. 


\section{FIGURE 2}

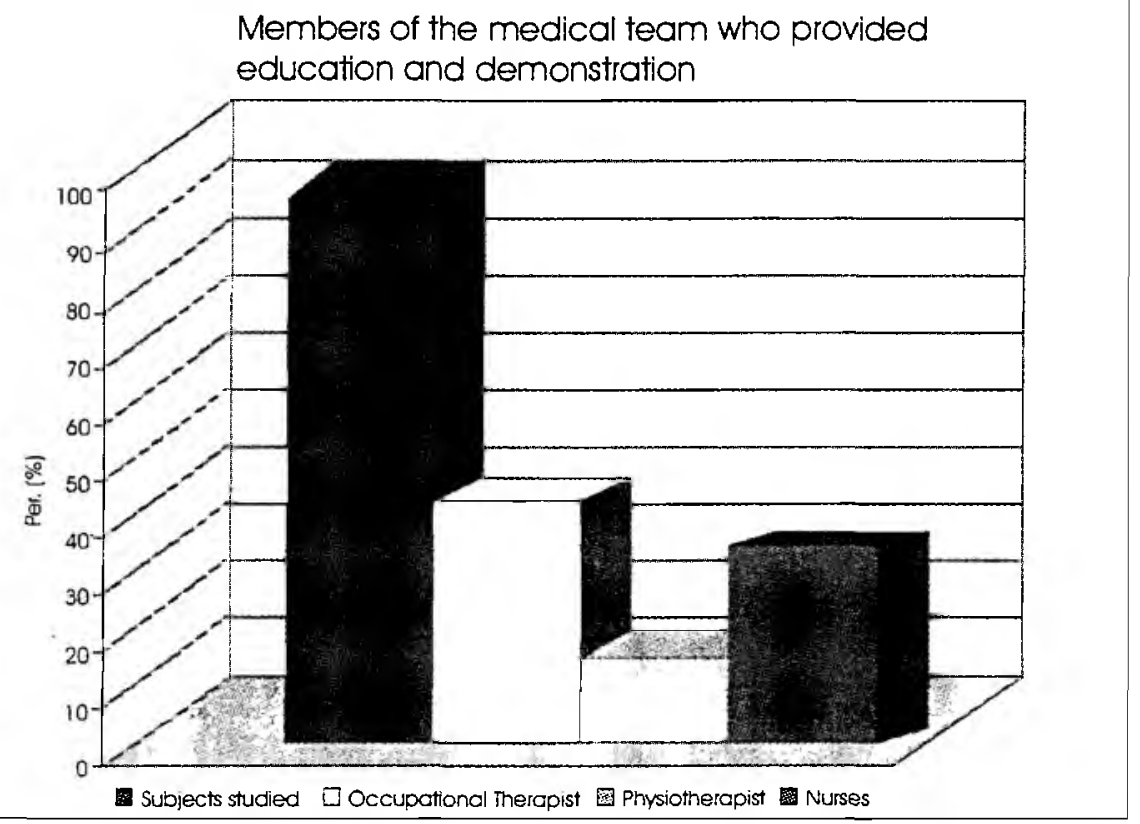

Fig 2: Members of the medical team who provided education and demonstration

following:

An information booklet that will include:

- Pre- and post-operative information to the patient.

- Applicable exercises and do's / don'ts.

- Setting the criteria for obtaining a prosthesis.

- Explaining the role of each member of the amputation team.

- Translating this information into other languages and distributing it to rural clinics.

\section{METHODS}

This investigation was done on the first
33 subjects who attended the amputation clinic at the PAH since the start of this study (1997/4/4). Only subjects with lower limb amputations were included in this study, while subjects who did not use the prescribed bandage for coning were excluded. Reasons for amputations included the following: tumors (5), trauma (11), diabetes (5), arteriosclerosis (12). Most amputation procedures were done at the PAH and referrals from other hospitals were as follows: private (3), Kalafong (4). All participants completed a consent form. Evaluations were done individually in an examination room, by the same physiotherapist.
The study included the following:

- A questionnaire to test the patient's knowledge and skills on amputation stump bandaging. The questionnaire was developed for this study, and pre-tested for clarity.

- A physical evaluation by the same physiotherapist on the effectiveness of the bandaging.

The criteria for effective stump bandaging included (Brady 1982):

- Bandage worn for more than 20 hours a day.

- Bandaging technique demonstrated by either the physiotherapist or occupational therapist and practised by the patient under supervision.

- The bandage washed every third day and the washing technique consisting of a gentle pressing out with the hands.

- Three bandages available at any given time.

- As the main reason for bandaging is shaping the stump for a prosthesis, most tension should be on the distal part of the stump.

- The physical application of the bandage included the following:

- Application to start distally.

- Bandage applied in a figure of eight.

- The bandage to end high enough in the groin for the above-knee amputee and five fingers above the patella for the below-knee amputation.

\section{RESULTS}

Twenty-one males and 12 females, aged between 23 years and 78 years participated in the study and the period of time after amputation was 6 weeks to 2 years.

1.1 Education to patients on bandaging of amputation stumps. (Figure 1)

Of the 33 subjects who participated in this study, only $16(49 \%)$ received previous education and demonstration on amputation stump bandaging. Seventeen $(52 \%)$ of the subjects had received no form of demonstration or education at all.

\subsection{Members of the medical team whoprovided education to the patients. (Figure 2)}

Sixteen subjects who received demonstration and education obtained their information from the following members of the team: Eight $(50 \%)$ received this information from an occupational therapist, two (13\%) from a physiotherapist and six (38\%) from a nurse. None of the subjects received written information on 
amputation stump bandaging as part of their education.

\subsection{Reasons for bandaging}

Eleven (69\%) of the 16 subjects knew that the main reason for amputation stump bandaging was coning, four $(25 \%)$ said that it was for pain control and 1 $(6 \%)$ for cosmetic reasons.

\subsection{Effectiveness of the bandaging. (Figure 3)}

Ten $(63 \%)$ of the 16 subjects wore the bandage for the prescribed 20 out of 24 hours a day.

All the subjects had more than one bandage and there was therefore no reason for a subject to go without stump bandaging at any stage.

Seven $(44 \%)$ continued the bandaging of the stump high enough.

Twelve $(75 \%)$ bandaged the stump in a figure of $8,14(88 \%)$ started with the bandaging distally and $10(63 \%)$ applied the bandage with enough pressure distally.

After individual analysis it was found that only $3(9 \%)$ subjects fitted all the criteria of effective stump bandaging.

\subsection{Handling of the bandage.}

Only $5(31 \%)$ of the 16 subjects knew how to wash the bandage correctly. To wash the bandage correctly is vital to the life span of these particular bandages, used for coning.

It is interesting to note that 2 out of the 16 subjects who had their amputations for just over 1 year still had fairly good knowledge and skill on amputation stump bandaging. Patients seemed to remember all aspects of this traumatic procedure, vividly.

Of further interest was the fact that more than half of the subjects referred from hospitals other than the PAH did not receive any form of education and demonstration.

It was alarming that seven out of 33 subjects did not receive any form of exercise and that two of these patients had to be sent back home with a flexion contracture of the hip.

\section{DISCUSSION}

From this study it is clear that there is a lack of knowledge and skill relating to bandaging amongst amputation patients and that this urgently needs to be rectified to ensure maximal functional outcomes (Esquenazi and Meier 1996). This can be achieved by involvement of all the members of the medical team in providing consistent education to the patient (Esquenazi and Meier 1996). Detailed instructions to the patient, followed by repetition and re-evaluation need to be emphasised. Individualising the education process with emphasis on reasons for bandaging need to be adressed
(Andrews 1996).

Ozyalcin and Sesli (1989) supports the findings from this survey. He stated that the problems encountered in developing countries are inadequate fitting of prosthesis and the lack of proper rehabilitation.

\section{CONCLUSION}

Physiotherapy is a vital part of the rehabilitation of amputees, which include assessment, post-operative exercises, transfers, bandaging techniques, education and gait training (Andrews 1996).

Stump and leg self-care procedures should be taught and reinforced throughout the rehabilitation period. Automatic integration of these routines into the amputee's adapted life-style only occurs after frequent and consistent repetition. Patients often have difficulty in comprehending and retaining information and instructions in the early stages of rehabilitation. However, simple written instructions can be provided to help ensure continued follow-up at home.

\section{REFERENCES}

Andrews KL 1996 Rehabilitation in limb deficiency. The geriatric amputee. Arch Phys Med Rehabil 77:S-14-S-17.2.

Brady WM 1982 Post-operative management of lower extremity amputees using Tubular Elastic Compression bandaging. Orthotics and Prosthetics 6:8-10.

Browse NL 1974 Amputation of the lower limb. Nursing Mirror June:63-65

Esquenazi A, Meier RH 1996 Rehabilitation in limb deficiency. Limb amputation. Arch Phys Med Rehabil 77:S-18-S-28.

Holliday PJ 1981 Non-prosthetic care. In JP Kostuik and R Gillespie, eds. Amputation surgery and rehabilitation: The Toronto experience. New York: Churchill Livingstone. 238-242.

Isherwood PA, Robertson JC, Rossi A 1975 Pressure measurements beneath below-knee amputation stump bandages: elastic bandaging, the Puddifoot dressing and a pneumatic bandaging technique compared. $\mathrm{Br}$. I Surg 62:982-986.

MacLean N, Fick GH 1994 The effect of semi-rigid dressings on below-knee amputations. Phys Ther 74:668-673.

O'Sullivan SB, Schmitz TJ 1988 Physical rehabilitation: Assessment and treatment 2ed. FA David Company. Philidelphia :385405 .

Ozyalcin H, Sesli E 1989 Temporary prosthetic fitting of below-knee amputation. Prosthetic and Orthotic Int. 13(2):86-9. Pinzur MS, Littoy F, Osterman H, Wafer D 1988 Early post-surgical prosthetic limb fitting in dysvascular below-knee amputees with a pre-fabricated tempory limb. Orthopeadics. 11(7):105I-3.

Spiro M, Roberts VC, Richarson JB 1980 The effect of externally applied pressure loads upon blood supply to the skin. $\mathrm{Br} \mathrm{Med}$ $J$ 1:719-723.

\section{QUESTIONNAIRE (SUMMARISED)}

1. How many hours (out of 24) do you wear the bandage?

between $0-8$ /between $8-12$ /more than 20

2. Was the bandaging technique shown to you?

no yes

3. Did you practice the bandaging under supervision?

yes no

4. How often do you wash the bandage?

every day every second day every third day once a week

5. Did you receive any written information on the bandaging technique? no yes

6. Who demonstrated the bandaging technique to you?

physiotherapist occupational therapist nurse other

7. Do you have more than one bandage?

no yes (how many?)

8. How do you wash the bandage? like washing clothes pressing it out with your hands use washing machine

9. What do you think is the main reason for bandaging?

for pain

looks better

shaping the stump for a prosthesis

keeping the stump warm other

10. Where must the most tension be after bandaging?

on the upper end of the stump on the lower end of the stump equally distributed

\section{Physical Evaluation}

1. Did the subject start distally with the application of the bandage?

2. Is the bandage applied in a figure of 8 ?

3. Does the bandage end high enough? 